\title{
Genetic basis and timing of a major mating system shift in Capsella
}

2

Jörg A. Bachmann ${ }^{1, \text {, }}$, Andrew Tedder ${ }^{1, \uparrow}$, Benjamin Laenen ${ }^{1, \uparrow}$, Marco Fracassetti ${ }^{1}$, Aurélie Désamoré $^{1}$, Clément Lafon-Placette ${ }^{2}$, Kim A. Steige $^{1}$, Caroline Callot ${ }^{3}$, William Marande ${ }^{3}$, Barbara Neuffer ${ }^{4}$, Hélène Bergès $^{3}$, Claudia Köhler ${ }^{2}$, Vincent Castric $^{5}$, Tanja Slotte ${ }^{1 *}$ ${ }^{1}$ Department of Ecology, Environment and Plant Sciences, Science for Life Laboratory, Stockholm University, SE-106 91 Stockholm, Sweden, ${ }^{2}$ Department of Plant Biology, Swedish University of Agricultural Sciences \& Linnean Center for Plant Biology, SE-750 07 Uppsala, Sweden ${ }^{3}$ Institut National de la Recherche Agronomique UPR 1258, Centre National des Ressources Génomiques Végétales, Castanet-Tolosan, France, ${ }^{4}$ Department of Botany, University of Osnabruck, Osnabruck, Germany, ${ }^{5}$ Univ. Lille, CNRS, UMR 8198 - Evo-Eco2 Paleo, F-59000 Lille, France

*Corresponding author: Email: tanja.slotte@su.se, Phone: +46-76-1252109

`These authors contributed equally to this work.

\section{Summary}

- A crucial step in the transition from outcrossing to self-fertilization is the loss of genetic self-incompatibility (SI). In the Brassicaceae, SI involves interaction of female and male specificity components, encoded by the genes $S R K$ and $S C R$ at the selfincompatibility locus ( $S$-locus). Theory predicts that $S$-linked mutations, and especially dominant mutations in $S C R$, are likely to contribute to loss of SI. However, few studies have investigated the contribution of dominant mutations to loss of SI in wild plant species. 
- Here, we investigate the genetic basis of loss of SI in the self-fertilizing crucifer species Capsella orientalis, by combining genetic mapping, long-read sequencing of complete $S$-haplotypes, gene expression analyses, and controlled crosses.

- We show that loss of SI in C. orientalis occurred less than 2.6 Mya and maps as a dominant trait to the $S$-locus. We identify a fixed frameshift deletion in the male specificity gene $S C R$ and confirm loss of male SI specificity. We further identify an $S$ linked small RNA that is predicted to cause dominance of self-compatibility.

- Our results agree with predictions on the contribution of dominant $S$-linked mutations to loss of SI, and thus provide new insights into the molecular basis of mating system transitions.

Keywords: Capsella, dominance modifier, long-read sequencing, parallel evolution, plant mating system shift, self-compatibility, S-locus, small RNA 


\section{Introduction}

The shift from outcrossing to self-fertilization is one of the most common evolutionary transitions in flowering plants (Darwin, 1876; Wright et al., 2013). This transition is favored when the benefits of reproductive assurance (Darwin, 1876; Pannell \& Barrett, 1998; Eckert et al., 2006) and the transmission advantage of selfing (Fisher, 1941) outweigh the cost of inbreeding depression (Charlesworth, 2006).

The transition to self-fertilization often involves breakdown of self-incompatibility (SI). SI systems allow plants to recognize and reject self pollen through the action of male and female specificity components and modifier loci (Takayama \& Isogai, 2005). In the Brassicaceae, SI is controlled by two tightly linked genes at the $S$-locus, the $S$-locus receptor kinase gene $S R K$ and $S C R$, which encode the female and male SI specificity determinants, respectively (de Nettancourt, 2001). SRK is a transmembrane serine-threonine receptor kinase located on the stigma surface (Stein et al., 1991; 1996). SCR is a small cysteine-rich protein that is deposited on the pollen coat and acts as a ligand to the SRK receptor (Schopfer et al., 1999; Takayama et al., 2001). Direct interaction between SRK and SCR from the same $S$ haplotype results in inhibition of pollen germination (Takasaki et al., 2000; Takayama et al., 2001; Ma et al., 2016) through a signaling cascade involving several proteins (Nasrallah \& Nasrallah 2014). This SI response prevents close inbreeding and promotes outcrossing. At the $S$-locus, recombination is suppressed and rare allele advantage maintains alleles with different specificities (Wright, 1939; Castric \& Vekemans, 2004; Vekemans et al., 2014). SI populations often harbor dozens of highly diverged $S$-haplotypes as a result of negative frequency-dependent selection (Mable et al., 2003; Guo et al., 2009). In the sporophytic Brassicaceae SI system, expression of a single $S$-specificity provides greater compatibility with other individuals (Schoen $\&$ Busch, 2009). Therefore, $S$-haplotypes often form a dominance hierarchy that determines which specificity is expressed in $S$-heterozygotes 
63 (Durand et al., 2014). At the pollen level, dominance is governed by dominance modifiers in the form of sRNAs expressed by dominant alleles. These sRNAs target sequence motifs specific to recessive alleles of $S C R$, resulting in transcriptional silencing (Tarutani et al., 2010; Durand et al., 2014).

Despite the advantages of outcrossing, SI has been lost repeatedly in many different lineages. There is a strong theoretical and empirical interest in the role of parallel molecular changes for repeated shifts to self-compatibility (SC) (Vekemans et al., 2014; Shimizu \& Tsuchimatsu, 2015). While the numerous genes that act as unlinked modifiers of SI potentially constitute a larger mutational target than the $S$-locus itself, theory predicts that mutations that result in degeneration of components of the $S$-locus should have an advantage (Porcher \& Lande, 2005). Theory further predicts that the probability of spread of mutations disrupting SI depends on whether they affect male or female SI function, or both functions jointly (Charlesworth \& Charlesworth, 1979). In particular, mutations that disrupt male specificity should have an advantage over those mutations that disrupt female specificity, because male specificity mutations can spread faster through both pollen and seeds (Uyenoyama et al., 2001; Tsuchimatsu \& Shimizu, 2013). Finally, dominant advantageous mutations should have a higher fixation probability in outcrossers, as expected from Haldane's sieve (Haldane 1927). However, dominant $S$-alleles typically have low population frequencies (Llaurens et al., 2008), resulting in a lower probability that SC mutations occur on dominant than on recessive alleles. While degeneration of male specificity has contributed to loss of SI in a few Brassicaceae species (Tsuchimatsu et al., 2010; 2012; Shimizu \& Tsuchimatsu, 2015) Chantha et al., 2013), more examples are needed. So far, few empirical studies of wild species have examined the contribution of dominant $S$-haplotypes to the loss of SI (but see Nasrallah et al. 2007). To understand the role of parallel molecular changes for recurrent loss of SI, identification of causal mutations is required. This has been a challenging 
task, due to the difficulty of sequencing the up to $110 \mathrm{~kb}$ long, highly polymorphic and repetitive $S$-locus. However, thanks to the advent of long-read sequencing, contiguous $S$ haplotypes can now be assembled with low error rates (Bachmann et al., 2018).

The crucifer genus Capsella is an emerging model for genomic studies of plant mating system evolution. In Capsella, SI is the ancestral state, as there is trans-specific shared $S$ locus polymorphism between the outcrossing SI species Capsella grandiflora and outcrossing SI Arabidopsis species (Guo et al., 2009). Nevertheless, SC has evolved repeatedly in Capsella, resulting in two self-compatible and highly selfing diploid species, Capsella rubella and Capsella orientalis, as well as the selfing allotetraploid Capsella bursa-pastoris, which formed by hybridization between $C$. orientalis and $C$. grandiflora accompanied by genome duplication (Douglas et al., 2015). These species also differ greatly in their geographical distributions, with C. bursa-pastoris having a nearly worldwide distribution, whereas $C$. rubella is mainly found in Central and Southern Europe, and C. orientalis has a distribution ranging from Eastern Europe to Central Asia (Hurka et al., 2012). Finally, the SI outcrosser C. grandiflora mainly occurs in northwestern Greece and Albania, and in northern Italy (Hurka et al., 2012).

In C. rubella, the transition to selfing has been intensely studied (Foxe et al., 2009; Guo et al., 2009; Slotte et al., 2013; Brandvain et al., 2013) and involved the fixation of a relatively dominant $S$-haplotype (Nasrallah et al., 2007; Guo et al., 2009; Paetsch et al. 2010) most likely within the past 100-170 ky (Slotte et al., 2013; Koenig et al., 2019). Knowledge on the mode, timing and demographics of the transition to selfing in C. rubella has provided an evolutionary context for the study of genomic (Gos et al., 2012; Slotte et al., 2013; Brandvain et al., 2013; Koenig et al., 2019), regulatory (Steige et al. 2015) and phenotypic (Slotte et al., 2012; Sicard et al., 2016) consequences of selfing. In contrast, we know little about the genetic basis and timing of loss of SI and transition to selfing in C. orientalis. Such 
113 information is important for proper interpretation of genomic studies of the effects of selfing

114 and can provide insights into the role of parallel molecular changes for convergent loss of SI.

115 Here, we combined genetic mapping, long-read sequencing of $S$-haplotypes,

116 controlled crosses, population genomic and expression analyses to investigate the loss of SI in

117 C. orientalis, with the specific aims to: 1) test whether loss of SI maps to the $S$-locus, 2)

118 identify candidate causal mutations for the loss of SI, 3) investigate the role of sRNA-based

119 dominance modifiers, and 4) estimate the timing of loss of SI in C. orientalis.

120

121 Materials and Methods

122 Plant material and growth conditions

123 We surface-sterilized seeds of Capsella orientalis Klokov, Capsella bursa-pastoris (L.)

124 Medik. and Capsella grandiflora (Fauché \& Chaub.) Boiss. (S1 Table, Supporting

125 Information), plated them on $1 / 2$ MS medium (Murashige and Skoog basal salt mixture,

126 Sigma-Aldrich Co. MI, USA) and stratified seeds at $2-4^{\circ} \mathrm{C}$ in the dark for two weeks. Plates

127 were then moved to controlled climate chambers $\left(16 \mathrm{~h}\right.$ light at $20^{\circ} \mathrm{C} / 8 \mathrm{~h}$ dark at $18{ }^{\circ} \mathrm{C}, 70 \%$

128 maximum humidity, $122 \mathrm{uE}$ light intensity). After one week, seedlings were transplanted to

129 soil in pots. For genotyping and whole-genome resequencing, leaf samples for DNA

130 extractions were collected from $>3$ week old plants and dried in silica gel. For bacterial

131 artificial chromosome (BAC) library construction, leaf samples were collected after $48 \mathrm{~h}$ dark

132 treatment and were immediately flash-frozen in liquid $\mathrm{N}_{2}$. For RNA extractions, mixed-stage

133 floral buds and leaf samples were collected in the middle of the light period and immediately

134 flash-frozen in liquid $\mathrm{N}_{2}$.

135

136 Genetic mapping of loss of SI in C. orientalis 
137 To test whether loss of SI mapped to the $S$-locus, we generated an interspecific $C$. orientalis $\times$

138 C. grandiflora F2 mapping population which segregated for SI/SC by crossing C. orientalis

139 accession Co2008-1 as seed parent to C. grandiflora accession Cg88.15 as pollen donor (S1

140 Table, Supporting Information). Because C. orientalis $\times$ C. grandiflora F1 seeds were aborted

141 prior to full development, generating viable F1 seeds required embryo rescue (Methods S1,

142 Supporting Information). F1 individuals were SC, and we collected F2 seeds from one

143 autonomously self-pollinated F1 individual. Our mapping population consisted of a total of

144350 F2 individuals. We extracted DNA from all F2 individuals using a Qiagen DNeasy kit

145 (Qiagen, Venlo, The Netherlands) and genotyped them at 998 SNPs at SciLifelab Stockholm

146 (Methods S1, Supporting Information).

147 We scored SI/SC in a total of $321 \mathrm{~F} 2$ individuals. SI/SC was scored as presence or

148 absence of silique formation on mature individuals. In addition, we assessed the success of 3-

1496 manual self-pollinations for 204 F2 individuals. In the case of a discrepancy between seed

150 set after manual self-pollination and silique formation after autonomous self-pollination, we

151 used the scoring based on manual self-pollination. To validate that the SI phenotype was due

152 to pollen tube growth arrest and the lack of seed development following self-pollination was

153 not due to e.g. inbreeding depression or later-acting genetic incompatibilities, we assessed

154 pollen tube growth in the pistil after manual self-pollination in a subset of $10 \mathrm{~F} 2$ individuals

155 scored as SI (Methods S1, Supporting Information).

156 We generated a linkage map and mapped quantitative trait loci (QTL) for SI/SC status

157 in R/Qtl (Broman et al., 2003). The final linkage map had 549 SNPs after removal of SNPs

158 with segregation distortion or redundant genotype information. We mapped QTL for SI/SC,

159 encoded as a binary trait, using interval mapping and the Haley \& Knott regression method

160 (Haley \& Knott, 1992) in intervals of $1 \mathrm{cM}$, based on 304 F2 individuals for which we had

161 both phenotype and genotype data. A 1\% genome-wide significance threshold was obtained 
by 1000 permutations and we estimated credible intervals of significant QTL as 1.5-LOD drop intervals. We estimated the additive allelic effect and dominance deviation using the R/Qtl effectscan function.

\section{Sequencing, assembly and annotation of the $S$-locus in Capsella}

To identify putative causal genetic changes responsible for loss of SI in C. orientalis, we conducted targeted sequencing and assembly of $S$-haplotypes by long-read sequencing of BAC clones containing the $S$-locus, as in Bachmann et al. (2018) (Methods S1, Supporting Information). We conducted targeted long-read sequencing and assembly of $S$-haplotypes of two SC C. orientalis accessions, four SC C. bursa-pastoris accessions and two SI $C$. grandiflora accessions. The two C. grandiflora $S$-haplotypes presented here were chosen from a larger set of $15 S$-haplotypes to represent the $C$. grandiflora $S$-haplotype segregating in our F2 population as well as a $C$. grandiflora $S$-haplotype from the same haplogroup as the $S$ haplotype of $C$. orientalis (see "Phylogenetic analyses of $S$-locus sequences" below; S1 Table, Supporting Information). In total, we here present eight full-length $S$-locus haplotypes obtained by targeted long-read sequencing (S1 Table and S2 Table, Supporting Information). As far as possible, we use the same accession designations as in previous studies. All accession information is listed in S1 Table, Supporting Information.

BAC clones were sequenced to high coverage (150-400x) using PacBio SMRT sequencing (S2 Table, Supporting Information). Short-read sequencing data for all BACs were generated on an Illumina MiSeq ( $>380 \mathrm{x}$; S2 Table, Supporting Information) and used for indel error correction as in Bachmann et al. (2018). All sequencing was done at the SciLifeLab National Genomics Infrastructure in Uppsala, Sweden. Sequences were assembled in HGAP3.0 (Chin et al., 2013), except for the S-haplotype of Cg88.15, for which Canu v.1.7 (Koren et al., 2017) was used. 
Augustus v3.2.3 (Stanke et al., 2004) and RepeatMasker v4.0.7;

Arabidopsis thaliana as a model prediction species and using protein homology data for $S R K$, U-box and ARK3 from Arabidopsis lyrata and Arabidopsis halleri. Due to high levels of

192 sequence diversity at the key $S$-locus genes $S R K$ and $S C R$, they were difficult to annotate automatically. Sequence similarity to known $S R K$ exon 1 sequences was used to accept candidate loci as $S R K$, while we used similarity to $A R K 3$ as a rejection criterion. To annotate $S C R$, we used a window-based approach to screen for the characteristic pattern of cysteine residues after translation of the DNA sequence in all three frames (Bachmann et al., 2018).

197 Using this approach, we identified a region highly similar to $A$. halleri $S C R$ in $S$-locus 198 haplotype $S 12$ (GenBank accession number KJ772374.1) in our C. orientalis S-locus BAC 199 sequences.

\section{Phylogenetic analyses of $S$-locus sequences}

202 To examine the phylogenetic placement of the $S$-haplotypes sequenced here, we used a dataset of Brassicaceae $S R K$ exon 1 and $A R K 3$ sequences downloaded from Genbank as described in Bachmann et al. (2018) and generated an alignment of SRK exon 1 sequences using the MAFFT v7.245 \& E-INS-I algorithm (Katoh et al., 2002), with manual curation in

206 SeaView v4.6 (Gouy et al., 2010). We generated a maximum likelihood phylogenetic tree 207 from the $S R K$ alignment with RaXMl v8.2.3. In this phylogeny, the $C$. grandiflora $S$ 208 haplotype from accession Cg2-2 clustered with the $S$-haplotypes of $C$. orientalis and the $C$. 209 orientalis-derived subgenome of C. bursa-pastoris (i.e. the C. bursa-pastoris B subgenome). 210 Due to the high sequence similarity ( $93.4 \%$ protein sequence identity at $S R K)$ of the Cg2-2 C. 211 grandiflora S-haplotype to A. halleri S12 (GenBank accession number KJ772374.1) we 
212 hereafter term this $S$-haplotype $C g S 12$. We assessed sequence conservation across the entire

$213 \sim 100 \mathrm{kbp} S$-locus by aligning $S$-locus sequences using LASTZ v1.03.54 (Harris, 2007) and

214 calculating pairwise sequence conservation in $250 \mathrm{bp}$ sliding windows.

\section{Candidate mutations for the loss of SI in $C$. orientalis}

217 To identify candidate causal mutations for the loss of SI in C. orientalis, we analyzed 218 sequence alignments of the two key $S$-locus genes $S R K$ and $S C R$, as well as the $S$-linked $U$ 219 box gene, which may act as a modifier of the SI response (Liu et al., 2007). Specifically, we 220 searched for major-effect variants resulting in frameshifts, premature stop codons or non221 consensus splice sites, present in sequences from the SC C. orientalis and/or in the SC $C$. 222 bursa-pastoris B subgenome, which is derived from C. orientalis (Douglas et al., 2015), but not in sequences from the same haplogroup found in the SI species $C$. grandiflora and $A$. halleri. For $S R K$ we identified nonsynonymous changes in hypervariable regions important for SRK specificity (Nishio \& Kusaba 2000; Kusaba et al. 1997), based on a protein sequence alignment of Capsella SRK with Brassica rapa SRK9 which represents a different haplogroup, but whose protein structure and interaction with SCR has been resolved recently (Ma et al., 2016).

Bioinformatic processing of RNAseq data and expression of $S$-locus genes in $C$. orientalis

231 RNAseq data were trimmed with Trimmomatic v.0.36 (Bolger et al. 2014) and reads mapped 232 using STAR v.2.2.1 (Dobin et al., 2013). For small RNA sequencing, we mapped reads of 233 length 18-27 nt using STAR v.2.2.1. Expression was quantified as RPKM (number of reads 234 per kb per million mapped reads; Mortazavi et al., 2008). To assess whether SRK, SCR and U-box were expressed in C. orientalis flower buds, 236 we generated RNAseq data from mixed-stage flower buds of two C. orientalis accessions (S1 
Table, Supporting Information) as previously described (Steige et al., 2017). For comparison, we also generated RNAseq data from leaf samples from the same individuals. Trimmed reads were mapped to a modified v1.0 reference $C$. rubella assembly (Slotte et al., 2013), where the $S$-locus region (scaffold_ 7 7523601:7562919) was masked and our $S$-locus assembly from $C$. orientalis Co1719/11 was added. We conducted qualitative RT-PCR with specific primers to $S C R$ in $C$. orientalis and $C$. grandiflora $C g S 12$, to assess the expression of $S C R$ in flower buds of both $C$. orientalis accessions, as well as in three $C$. grandiflora individuals harboring CgS12 (Methods S1, Supporting Information).

\section{Assessing the functionality of $C$. orientalis SCR by interspecific crosses}

247 We performed controlled crosses to verify that $C$. grandiflora $C g S 12$ conferred SI, and to assess the functionality of SCR in $C$. orientalis. To verify functional SI in C. grandiflora carrying $\mathrm{CgS12}$, we performed a total of 24 manual self-pollinations of four C. grandiflora individuals carrying the $C g S 12 S$-haplotype. While the identity of the other $S$-haplotype in

251 these individuals is unknown and we were unable to identify it using PCR-based screening, 252 we verified expression of $C g S C R 12$, indicating that the other $S$-allele is not dominant over 253 CgS12 at the pollen level. We assessed the success of manual self-pollination of $C$. orientalis by performing 6-12 manual self-pollinations of each of three accessions (Table S1,

255 Supporting Information). To assess whether $C$. orientalis SCR is functional, we crossed $C$. 256 grandiflora harboring $C g S 12$ as a seed parent to $C$. orientalis as a pollen donor. We 257 performed a total of 144 crosses of this type, with three different $C$. orientalis accessions as 258 pollen donors and six different $C g S 12$-carrying $C$. grandiflora individuals as seed parents 259 (Table S1, Supporting Information). If C. orientalis SCR is functional, and provided that $260 C g S 12 S R K$ is expressed, then we expect this cross to be incompatible, whereas if $C$. 261 orientalis SCR is nonfunctional, the cross should be compatible. The reciprocal cross of the 
same individuals was also carried out with the same accessions (total 104 crosses of this

263 284 allele. type), to test whether female SI specificity is functional in C. orientalis. Finally, we performed 12 crosses of $C$. grandiflora harboring other $S$-haplotypes to $C$. grandiflora harboring $C g S 12$, and 12 to $C$. orientalis. These crosses are expected to be successful. We observed pollen tube growth in the pistil 12 hours after pollination. Pistils were fixed in EtOH: acetic acid 9:1 for $>2$ hours, softened in $1 \mathrm{~N} \mathrm{NaOH} 60^{\circ} \mathrm{C}$ for 20 minutes and stained with $0.01 \%$ decolorised aniline blue in $2 \%$ solution of $\mathrm{K}_{3} \mathrm{P} \mathrm{O}_{4}$ for 2 hours. Pollen tubes were visualised by mounting pistils on a microscope slide which was examined under an epifluorescence microscope (Zeiss Axiovert 200M). We compared the number of pollen tubes among different types of crosses using a Kruskal-Wallis test.

\section{The role of small RNA-based dominance modifiers for dominance of SC}

To test whether dominant expression of SC could be mediated by small RNA-based dominance modifiers, we conducted additional sequence and expression analyses, using a strategy similar to that of Nasrallah et al. (2007). We identified a region in our C. orientalis $S$ haplotypes with high sequence similarity $(91.3 \%)$ to the A. halleri S12 small RNA precursor Ah12mirS3 identified previously (Durand et al., 2014). We generated small RNA and RNA sequencing data from flower buds of $19 \mathrm{~F} 2$ s, representing all three $S$-locus genotypes in our F2 mapping population (12 heterozygotes, 4 and 3 individuals homozygous for the $C$.

orientalis or the C. grandiflora $S$-haplotype, respectively). We quantified expression of sRNAs in the Ah12mirS3-like sRNA precursor region (hereafter termed ComirS3 sRNAs) and tested whether ComirS3 sRNAs were expressed specifically in F2s with a C. orientalis $S$ -

To test whether $C$. grandiflora $S C R$ was repressed in F2s heterozygous at the $S$-locus we quantified the expression of $C$. orientalis and C. grandiflora SCR in our F2s. We mapped 
F2 RNAseq reads from flower buds to a modified C. rubella reference containing both the Co1719/11 S-haplotype and the C. grandiflora Cg88.15 S-haplotype segregating in our F2 population, and quantified the expression of C. orientalis and C. grandiflora SCR in all three genotypes, respectively.

To identify targets of ComirS3 small RNAs we took all 18-27 nt ComirS3 sRNAs and searched for small RNA targets within $1 \mathrm{~kb}$ of $S C R$ of the $C$. grandiflora $\mathrm{Cg} 88.15 S$ haplotype. Small RNA targets were identified using a Smith \& Waterman algorithm (Smith \& Waterman, 1981) with scoring matrix: match $=01$, mismatch $=-1$, gap $=-2, \mathrm{G}: \mathrm{U}$ wobble $=-0.5$ as previously described (Durand et al., 2014).

\section{Timing of loss of SI in C. orientalis}

To assess whether major-effect mutations at the $S$-locus were fixed in $C$. orientalis, we analyzed whole-genome resequencing data from additional $C$. orientalis accessions, in total covering 30 accessions from 18 populations and including publicly available $C$. orientalis genome resequencing data (Douglas et al., 2015; Huang et al., 2018; Koenig et al., 2019) (Table S1, Supporting Information). We mapped trimmed data to a C. rubella reference modified to include the $C$. orientalis haplotype of accession Co1719/11 using BWA-MEM (Li, 2013) and used GATK 3.8 (McKenna et al., 2010; DePristo et al., 2011; Van der Auwera et al., 2013) Unified Genotyper with the option --output_mode

EMIT_ALL_CONFIDENT_SITES to call all sites. We filtered sites following GATK recommended hard filtering with the following parameters; QD $<2.0\|\mathrm{FS}>60.0\| \mathrm{MQ}<$ 40.0 || MQRankSum $<-12.5||$ ReadPosRankSum $<-8.0$. We required a minimum read depth of 15 and a maximum of 200. Finally, we scored the presence or absence of major-effect mutations at the $S$-locus in our samples. Because $C$. orientalis is highly homozygous, SC, and has low levels of polymorphism genome-wide (Douglas et al., 2015), this approach is 
expected to work well, as long as a $C$. orientalis $S$-haplotype is included in the reference genome.

We used a strategy similar to that in Guo et al. (2009) to estimate a lower and upper bound of the timing of the loss of SI in C. orientalis. We obtained a lower bound for the

316 timing of the loss of SI by estimating the time to the most recent common ancestor (TMRCA) based on full-length $C$. orientalis and $C$. bursa-pastoris B $S$-locus sequences. Genome-wide haplotype sharing between $C$. orientalis and the $C$. bursa-pastoris $\mathrm{B}$ subgenome suggests that the ancestor of $C$. orientalis that contributed to formation of C. bursa-pastoris was selfcompatible (Douglas et al., 2015) and including C. bursa-pastoris B sequences can thus increase the precision of our estimates. To obtain an upper bound for the timing of the loss of SI we estimated the TMRCA for C. orientalis, C. bursa-pastoris B and C. grandiflora CgS12. For analyses of the timing of loss of SI, our final alignment contained $37 \mathrm{~S}$-locus sequences including the $C$. grandiflora ancestral $S$-haplotype (CgS12), 4 C. bursa-pastoris subgenome B $S$-haplotypes and $S$-haplotype data for 32 C. orientalis individuals (Supporting Information). Sequences were aligned using block alignment using Muscle v.3.8.31 (Edgar 2004) as implemented in AliView v.1.20 (Larsson 2014). The total length of the $S$-locus alignment was 33,485 bp, 22,689 bp had indels in at least one sequence, 9,835 sites were invariant and 876 sites were polymorphic. The alignment was partitioned into coding and non-coding regions and sites with indels and missing data were pruned in further analysis. We estimated the timing of the splits between C. grandiflora, C. bursa-pastoris and $C$. 332 orientalis using a strict molecular clock in a Bayesian framework in BEAST2 (Bouckaert et al. 2014). We used a fixed clock rate assuming a mutation rate of $7 \times 10^{-9}$ substitutions per site

334 per generation (Ossowski et al., 2010) and a generation time of one year. We ran both a model with exponential changes in population size and a model with a constant population size, and 336 compared models using Akaike's information criterion through Markov chain Monte Carlo, 
AICM (Baele et al. 2012) (Methods S1, Supporting Information). We ran two chains of 10 millions generations sampled every 1000 generations and checked convergence by visual inspection of the log-likelihood profile and assuring an effective sample size (ESS) value above 200. The posterior distribution of trees was used to build a maximum clade credibility tree and estimate node age and 95\% confidence interval using TreeAnnotator (Drummond et

342 al. 2012).

\section{Results}

\section{SC maps to the $S$-locus as a dominant trait}

We first asked whether loss of SI in $C$. orientalis maps to the canonical Brassicaceae $S$-locus. We therefore generated an F2 mapping population by crossing SC C. orientalis to a SI $C$. grandiflora accession. Interspecific $\mathrm{F} 1$ individuals were $\mathrm{SC}$, indicating that $\mathrm{SC}$ is dominant.

349 Our F2 mapping population segregated for SC, and we detected a single, significant $(P<0.001)$ quantitative trait locus $(\mathrm{QTL})$ for this trait, based on $304 \mathrm{~F} 2$ individuals genotyped at 549 markers (Fig. 1a; Fig. S1, Supporting Information). The credible interval for this QTL includes the $S$-locus on chromosome 7 (Fig. 1a), and SC was dominant over SI (Fig. 1b). SC in $C$. orientalis thus maps as a dominant trait to a region encompassing the $S$-locus.

\section{Sequencing the S-haplotype of $C$. orientalis and a highly similar but functional $S$ -}

357 We next sought to identify candidate causal loss-of-function mutations at the C. orientalis $S$ -

358 locus. For this purpose, we assembled full-length $S$-haplotype sequences of two $C$. orientalis 359 accessions based on long-read sequencing of BACs (Tables S1-S2, Supporting Information). 360 To facilitate identification of candidate mutations for the loss of SI, it is beneficial to be able 361 to contrast functional and non-functional $S$-haplotypes that belong to the same $S$-haplogroup 
and ancestrally shared the same SI specificity. Here, we identified and sequenced a functional C. grandiflora S-haplotype (for details, see Materials and Methods), which had $98.3 \%$ protein sequence identity at $S R K$ to that of $C$. orientalis (Fig. 2a-c, Table S3, Supporting Information). According to criteria used in outcrossing Arabidopsis species (Castric et al., 2008; Chantha et al., 2013), this C. grandiflora haplotype is expected to represent the same SI specificity as that of $C$. orientalis. This $C$. grandiflora $S$-haplotype is also similar $(93.4 \%$ protein sequence identity at $S R K$ ) to the functional Arabidopsis halleri S12 haplotype (Durand et al., 2014) (Fig. 2a-b, Fig. S2, Supporting Information), and we therefore designate it CgS12. Sequence similarity between $C g S 12$ and $C$. orientalis is not limited to $S R K$, as other $S$-linked genes showed the same phylogenetic topology as those for $S R K$ (Fig. 2b), and there were peaks of sequence conservation between $C g S 12$ and $C$. orientalis in both genic and intergenic parts of the $S$-locus (Fig. 2c). We verified that $C$. grandiflora individuals with CgS12 expressed $C g S C R 12$ and were SI by scoring pollen tube germination after controlled self-pollination (Fig. 3a, Table S4, Fig. S3-S5, Supporting Information).

\section{A frameshift deletion in the male specificity gene $S C R$ is fixed in $C$. orientalis}

By comparing $S$-haplotype sequences from $C$. orientalis (SC) to C. grandiflora $C g S 12$ and $A$. halleri S12 (both SI), we identified a single-base frameshift deletion in the SCR coding sequence of C. orientalis (Fig. 2d). This frameshift is predicted to result in loss of 5 out of 8 conserved cysteine residues essential to the function of SCR (Fig. 2e), likely resulting in loss

382 of male specificity. To assess whether the deletion was fixed in C. orientalis, as we would expect for mutations that spread early during the transition to selfing, we analyzed wholegenome resequencing data from additional C. orientalis accessions (S1 Table, Supporting Information). We found that the $S C R$ frameshift deletion was fixed across 32 samples of $C$. orientalis from 18 populations, consistent with expectations if the deletion was fixed in 
association with the loss of SI. The same deletion was found in SCR of the C. bursa-pastoris

388 B subgenome, which is derived from $C$. orientalis (Fig. 2d, Fig. 2e). This suggests that $C$. orientalis was self-compatible when it contributed to the origin of the allotetraploid C. bursapastoris.

In contrast to $S C R$, we observed no major loss-of-function mutations in C. orientalis $S R K$ or at the $S$-linked $U$-box gene, which may modify the female SI response (Liu et al., 2007). There were two nonsynonymous substitutions in C. orientalis $S R K$ that were likely located within hypervariable regions of SRK (Fig. S6, Supporting Information). However, without resolving the detailed protein structure of the $\mathrm{CgS} 12 \mathrm{SRK} / \mathrm{SCR}$ complex the exact consequences of these nonsynonymous changes cannot be determined. Finally, SRK, U-box and the truncated version of SCR are all expressed in flower buds of $C$. orientalis (Table S4, Fig. S5, Supporting Information) and we currently cannot rule out that more subtle changes to their sequence or expression affect their function.

\section{Assessment of SI specificity}

402 To assess whether male SI specificity is degenerated in C. orientalis, as we expect if SCR is nonfunctional, we crossed $C$. orientalis to $C$. grandiflora individuals harboring $C g S 12$, which likely ancestrally shared the same SI specificity (Fig. 2). As expected if the frameshift deletion impaired the function of $S C R$, pollen from $C$. orientalis successfully germinated on

406 the stigma of $C$. grandiflora individuals harboring $C g S 12$ (Fig. 3, Fig. S3-S4, Supporting 407 Information). However, we also found evidence for degeneration of female specificity in $C$. 408 orientalis, as pollen from $C$. grandiflora harboring $C g S 12$ germinated on the $C$. orientalis 409 stigma (Fig. 3; Fig. S3-S4, Supporting Information). Similar results were obtained for crosses 410 with C. orientalis accessions from three different populations (Fig. S4, Supporting 411 Information). 


\section{A conserved $S$-linked sRNA is associated with dominant expression of $C$. orientalis $S C R$}

414 Under most circumstances, loss of function mutations are predicted to be recessive, as a single 415 copy of a functional allele is often sufficient to result in a complete phenotype (Kacser \& 416 Burns, 1981). Here, SC is associated with a frameshift deletion at SCR, yet it is dominant in 417 our F2s. Hence, we investigated whether the small RNA-based mechanism that governs 418 dominance hierarchies among S-alleles in Arabidopsis (Durand et al., 2014) could also 419 explain dominance of SC in our case. Specifically, if the C. orientalis $S$-haplotype encodes a 420 trans-acting sRNA that represses expression of C. grandiflora SCR in S-locus heterozygotes, 421 SC could be dominant even if it is due to a loss of function mutation in C. orientalis SCR. In $A$. halleri, the $S 12$ haplotype belongs to the second most dominant class of $S$-alleles and harbors an S-linked sRNA-based dominance modifier termed Ah12mirS3 (Durand et al., 2014). In C. orientalis, we found the corresponding mirS3 sRNA precursor region to be conserved (91.3\% sequence identity) (Fig. 4a, Fig. S2, Supporting Information). The region harboring the mirS3 sRNA precursor was also conserved between C. grandiflora $C g S 12$ and C. orientalis (Fig. 2c). To assess whether expression of C. orientalis Ah12mirS3-like sRNA (ComirS3) was associated with repression of the $C$. grandiflora SCR allele passed on in our cross through the F1 plant, we sequenced and assembled the $C$. grandiflora $S$-haplotype segregating in our F2 population, and analyzed $S C R$ and sRNA expression in flower buds of 19 F2s representing all three possible $S$-locus genotypes. We detected expression of ComirS3 sRNAs (Fig. 4a) in F2s harboring the $C$. orientalis $S$-haplotype, but not in $C$. grandiflora $S$ homozygotes (Fig. 4b). The most abundant ComirS3 sRNA was highly similar to the Ah12mirS3 sRNA and had a predicted target within the intron of C. grandiflora SCR allele (Fig. 4c). The sRNA-target affinity was similar to that of functional Arabidopsis dominance modifiers (Durand et al., 2014; Burghraeve et al., 2018). As expected if ComirS3 sRNAs 
silence C. grandiflora SCR, C. grandiflora $S C R$ was specifically downregulated in $S$-locus heterozygotes (Fig. 4d). Our F2 $S$-locus heterozygotes thus only express the truncated $C$. orientalis $S C R$ at detectable levels. These results are consistent with $S$-linked sRNAs conferring dominance of the SC C. orientalis $S$-haplotype through transcriptional silencing of recessive $S C R$ alleles.

\section{Timing of loss of SI in C. orientalis}

The timing of loss of SI can be estimated based on polymorphism accumulated at the $S$-locus after loss of SI (Guo et al., 2009). We analyzed 37 full-length $S$-locus sequences and estimated an upper bound for the timing of loss of SI in C. orientalis as the time to the most recent common ancestor (TMRCA) of C. orientalis, C. bursa-pastoris B and C. grandiflora CgS12 S-haplotypes. Based on these analyses, we infer an upper bound of the timing loss of SI in C. orientalis at 2.6 Mya (2.2-2.9 Mya, 95\% CI) and a lower bound at 70 kya (50-100 kya, 95\% CI) (Fig. 5, Table S5, Supporting Information) under an exponential population size change model. Very similar estimates were obtained under a constant population size model and after subsampling the $C$. orientalis accessions to obtain a scattered sample (S5 Table, Supporting Information). Our timing estimates thus appear to be robust to sampling strategy and assumptions regarding population size changes.

\section{Discussion}

Here, we show that loss of SI in $C$. orientalis maps as a dominant trait to the $S$-locus. This result is consistent with the theoretical prediction that $S$-linked mutations should often contribute to the loss of SI (Porcher \& Lande, 2005). We identify candidate mutations for the loss of SI, including a frameshift deletion in the male specificity gene $S C R$. Our finding that $\mathrm{SC}$ is dominant agrees with Haldane's prediction that dominant alleles enjoy a higher fixation 
probability in outcrossers (Haldane 1927). Finally, we identify an sRNA that could be responsible for dominance of SC and that is conserved between Capsella and Arabidopsis halleri.

Theory predicts that mutations that disrupt male SI specificity should be more strongly selected for during the transition to selfing than those that disrupt female SI specificity (Uyenoyama et al., 2001; Busch \& Schoen, 2008; Tsuchimatsu \& Shimizu, 2013). Indeed, mutations that disrupt male SI specificity should have an advantage both when spreading through seeds and pollen, because they avoid recognition and rejection when they spread through outcross pollen (Uyenoyama et al., 2001; Busch \& Schoen, 2008; Tsuchimatsu \& Shimizu, 2013). In contrast, mutations that disrupt female specificity only have an advantage over those that disrupt male specificity when there is pollen limitation of seed set, i.e. reduced reproductive success due to inadequate quantity or quality of pollen (Uyenoyama et al., 2001; Busch \& Schoen, 2008; Tsuchimatsu \& Shimizu, 2013). The C. orientalis SCR deletion is expected to lead to the loss of 5 of 8 conserved cysteine residues in the SCR protein, which could cause loss of male SI specificity. The $S C R$ deletion was fixed in a broad sample of $C$. orientalis, as we would expect if it arose early during the transition to selfing. It was also found in the allopolyploid C. bursa-pastoris, suggesting that the shift to SC in C. orientalis predated the origin of C. bursa-pastoris. Through crosses between C. orientalis and $C$. grandiflora individuals harboring highly similar $S$-haplotypes, we confirmed that male SI specificity was lost in $C$. orientalis, as the pollen of $C$. orientalis germinated on the stigma of individuals harboring the highly similar but functional $C g S 12$ haplotype. However, we cannot strictly rule out a contribution of $S$-linked mutations that disrupt female SI specificity to the loss of SI in C. orientalis, as our controlled crosses indicated that female SI specificity was also impaired in C. orientalis. We identified two fixed nonsynonymous substitutions in likely functionally important regions of $S R K$ that might have contributed to the breakdown of 
female SI specificity in C. orientalis. However, without further work it is difficult to predict the functional consequences of these nonsynonymous substitutions. One scenario that would be consistent with our crossing results is one where the C. grandiflora CgS12 S-haplotype represents a different SI specificity than that of $C$. orientalis. Due to the very high sequence 491 similarity between these $S$-haplotypes, we consider this unlikely. Instead, we believe that our 492 crossing results illustrate a general challenge for studies that aim to identify causal changes for the loss of SI. Indeed, after SI has been lost, additional mutations that impair the function of $S$-locus genes can accumulate without cost, unless there are pleiotropic constraints. Ancestral reconstruction would be the only way to tease apart the role of these individual mutations to the breakdown of SI (Tsuchimatsu et al., 2010). Information on the timing of loss of SI is currently available for less than a handful of Brassicaceae systems (e.g. Guo et al. 2009; Busch et al. 2011; Tsuchimatsu et al., 2012). Accurately estimating bounds for the timing of loss of SI is challenging, as it requires identifying and sequencing shared $S$-haplotypes in closely related SI and SC species. Here, we 501 identify shared S-haplotypes in the SI C. grandiflora and the SC C. orientalis. We estimate that the loss of SI in C. orientalis occurred between 2.6 Mya and $70 \mathrm{kya}$, based on TMRCA analyses of full-length $S$-haplotypes. While our estimates cover a broad range of times, we argue that the most likely estimate of the timing of loss of SI is probably closer to the upper bound, 2.6 Mya. For instance, in comparison to the recently derived selfer C. rubella, $C$. orientalis has strongly reduced genome-wide polymorphism levels (Douglas et al., 2015;

507 Koenig et al. 2019), shows increased reproductive isolation through endosperm development 508 defects in crosses to C. grandiflora (Lafon-Placette et al., 2018), and possibly exhibits a lower 509 genomic content of transposable elements ( $\AA$ gren et al., 2014). An older origin of selfing in

510 C. orientalis than in C. rubella would be compatible with these findings, as selfing is

511 expected to result in reduced polymorphism genome-wide and affect TE content (Wright et 
al., 2013). While the shift to SC was clearly independent in C. orientalis and C. rubella, which harbor different $S$-haplotypes (Fig. 2a), both transitions involved fixation of a single $S$ haplotype (Guo et al., 2009; Slotte et al., 2012). These scenarios contrast with the situation in A. thaliana, where multiple $S$-haplogroups are still segregating (Durvasula et al., 2017;

516 Tsuchimatsu et al., 2017). Our study thus contributes to an improved understanding of the 517 timing and mode of loss of SI in a system that is widely used for genomic studies. Population geneticists have long predicted that dominant beneficial mutations should

519 have a higher fixation probability than recessive ones (Haldane, 1927), a phenomenon termed 520 "Haldane's sieve". Our finding that SC is dominant over SI is consistent with this prediction, 521 and agrees with results for several other wild Brassicaceae species (e.g. L. alabamica; Busch 522 et al., 2011, A. kamchatica; Tsuchimatsu et al., 2012, C. rubella; Nasrallah et al., 2007; Slotte 523 et al., 2012). However, not all transitions involve dominant $S$-haplotypes, and for instance in A. lyrata, a transition involving a recessive loss of SI has recently been documented (Mable et al., 2017). Our results further suggest that a small RNA-based mechanism could explain

526 dominance of SC. If this is the case, dominance of the SC phenotype will depend on the exact 527 combination of $S$-alleles and their position in the dominance hierarchy. Interestingly, in both 528 C. orientalis and C. rubella, SC is linked to relatively dominant $S$-haplotypes. Taken together, 529 these findings suggest that dominant SC mutations on average have an advantage over 530 recessive mutations, at least early during the transition to selfing. Thus, the lower population 531 frequencies or higher $S$-linked load (Llaurens et al., 2009) of dominant $S$-alleles do not 532 prevent mutations in such alleles from contributing to recurrent loss of SI.

\section{Acknowledgements}

535 We thank Timothy Paape and Matthew Hartfield for helpful discussion, Daniel Koenig and 536 Detlef Weigel for making C. orientalis resequencing data publicly available prior to 
publication, Cindy Canton for help with plant care, Olga Vinnere Pettersson and Christian Tellgren-Roth for help with long-read sequencing and BAC assembly. The authors acknowledge support from the National Genomics Infrastructure (NGI) / Uppsala Genome Center / SNP\&SEQ Technology Platform and UPPMAX for assistance in massive parallel 541 sequencing and computational infrastructure. Work at Uppsala Genome Center is funded by

542 RFI / VR and Science for Life Laboratory, Sweden. The SNP\&SEQ Platform is supported by 543 the Swedish Research Council and the Knut and Alice Wallenberg Foundation. V.C.

544 acknowledges support by a grant from the European Research Council (NOVEL project, 545 grant \#648321). The authors thank the French Ministère de l'Enseignement Supérieur et de la

546 Recherche, the Hauts de France Region and the European Funds for Regional Economical

547 Development for their financial support to this project. This work was supported by a grant 548 from the Swedish Research Council (grant \#D0432001) and by a grant from the Science for 549 Life Laboratory, Swedish Biodiversity Program to T.S. The Swedish Biodiversity Program is 550 supported by the Knut and Alice Wallenberg Foundation.

\section{Author contributions}

T.S. designed the experiments. J.B., A.T., C.L.-P., K.A.S., C.C. and W.M. performed the 554 experiments, J.B., A.T. and A.D. generated the data. J.B. analyzed sRNA expression and targets, A.T. analyzed and annotated $S$-locus BACs, B.L. analyzed and annotated $S$-locus BACs and performed BEAST analyses, M.F. analyzed QTL mapping and expression data, B.N. contributed reagents/materials/analysis tools, and A.D. generated full-length $S$-locus 558 alignments, H.B. supervised the work of C.C. and W.M., C.K. supervised the work of C.L.-P., 559 V.C. supervised the work of J.B, T.S. supervised the work of A.D., A.T., B.L., J.B., K.A.S. and M.F. All authors contributed to the writing of the paper. 


\section{References}

563 Bachmann JA, Tedder A, Laenen B, Steige KA, Slotte T. 2018. Targeted long-read

564 sequencing of a locus under long-term balancing selection in Capsella. G3 8: 1327-1333.

565 Baele G, Li WLS, Drummond AJ, Suchard MA, Lemey P. 2012. Accurate model selection

566 of relaxed molecular clocks in Bayesian phylogenetics. Molecular Biology and

567 Evolution 30:239-243.

568 Bolger AM, Lohse M, Usadel B. 2014. Trimmomatic: a flexible trimmer for Illumina

569 sequence data. Bioinformatics. 30:2114-2120.

570 Bouckaert R, Heled J, Kühnert D, Vaughan T, Wu C-H, Xie D, Suchard MA, Rambaut

571 A, Drummond AJ. 2014. BEAST 2: a software platform for Bayesian evolutionary analysis.

572 PLoS Computational Biology 10:e1003537.

573 Brandvain Y, Slotte T, Hazzouri KM, Wright SI, Coop G. 2013. Genomic identification of

574 founding haplotypes reveals the history of the selfing species Capsella rubella. PLos Genetics 575 9: e1003754.

576 Broman KW, Wu H, Sen S, Churchill GA. 2003. R/qtl: QTL mapping in experimental 577 crosses. Bioinformatics 19: 889-890.

578 Busch JW, Schoen DJ. 2008. The evolution of self-incompatibility when mates are limiting.

579 Trends in Plant Science 13: 128-136.

580 Busch JW, Joly S, Schoen DJ. 2011. Demographic signatures accompanying the evolution

581 of selfing in Leavenworthia alabamica. Molecular Biology and Evolution 28: 1717-1729.

582 Burghgraeve N, Simon S, Barral S, Fobis-Loisy I, Holl AC, Ponitzki C, Schmitt C,

583 Vekemans X, Castric V. 2018. Base-pairing requirements for small RNA-mediated gene 
584 silencing of recessive self-incompatibility alleles in Arabidopsis halleri. BioRxiv doi:

Castric V, Vekemans X. 2004. Plant self-incompatibility in natural populations: a critical assessment of recent theoretical and empirical advances. Molecular Ecology 13: 2873-2889.

Castric V, Bechsgaard J, Schierup MH, Vekemans X. 2008. Repeated adaptive introgression at a gene under multiallelic balancing selection. PLoS Genetics 4: e1000168.

591 evolution of a self-incompatibility locus in the Brassicaceae genus Leavenworthia. PLoS

592 Biology 11:e1001560.

Charlesworth D. 2006. Evolution of plant breeding systems. Current Biology 16: R726-35.

594 Charlesworth D. \& Charlesworth B. 1979. The evolution and breakdown of $S$-allele 595 systems. Heredity. 43: 41-55.

596 Chin C-S, Alexander DH, Marks P, Klammer AA, Drake J, Heiner C, Clum A, 597 Copeland A, Huddleston J, Eichler EE, et al. 2013. Nonhybrid, finished microbial genome 598 assemblies from long-read SMRT sequencing data. Nature Methods. 10: 563-569.

599 Darwin C. 1876. The effects of cross and self fertilisation in the vegetable kingdom. London: 600 John Murray.

601 Dobin A, Davis CA, Schlesinger F, Drenkow J, Zaleski C, Jha S, Batut P, Chaisson M, 602 Gingeras TR. 2013. STAR: ultrafast universal RNA-seq aligner. Bioinformatics. 29: 15-21.

603 de Nettancourt D. 2001. Incompatibility and Incongruity in Wild and Cultivated Plants.

604 Berlin: Springer. 
DePristo MA, Banks E, Poplin R, Garimella KV, Maguire JR, Hartl C, Philippakis AA, del Angel G, Rivas MA, Hanna M, et al. 2011. A framework for variation discovery and genotyping using next-generation DNA sequencing data. Nature Genetics 43: 491-498.

Douglas GM, Gos G, Steige KA, Salcedo A, Holm K, Josephs EB, Arunkumar R, Agren JA, Hazzouri KM, Wang W, et al. 2015. Hybrid origins and the earliest stages of diploidization in the highly successful recent polyploid Capsella bursa-pastoris. Proceedings of the National Academy of Sciences of the USA 112: 2806-2811.

Drummond AJ, Suchard MA, Xie D, Rambaut A. 2012. Bayesian phylogenetics with BEAUti and the BEAST 1.7. Molecular Biology and Evolution 29:1969-1973.

\section{Durand E, Méheust R, Soucaze M, Goubet PM, Gallina S, Poux C, Fobis-Loisy I,}

Guillon E, Gaude T, Sarazin A, et al. 2014. Dominance hierarchy arising from the evolution of a complex small RNA regulatory network. Science 346: 1200-1205.

Edgar RC. 2004. MUSCLE: multiple sequence alignment with high accuracy and high throughput. Nucleic Acids Research 32:1792-1797.

Durvasula A, Fulgione A, Gutaker RM, Alacakaptan SI, Flood PJ, Neto C, Tsuchimatsu T, Burbano HA, Picó FX, Alonso-Blanco C, et al. 2017. African genomes illuminate the early history and transition to selfing in Arabidopsis thaliana. Proceedings of the National Academy of Sciences of the USA 114: 5213-5218.

Eckert C, Samis K, Dart S. 2006. Reproductive assurance and the evolution of uniparental reproduction in flowering plants. In: Harder L, Barrett S, eds. Ecology and evolution of flowers. Oxford: Oxford University Press.

Fisher RA. 1941. Average excess and average effect of a gene substitution. Annals of Human 
628 Foxe JP, Slotte T, Stahl EA, Neuffer B, Hurka H, Wright SI. 2009. Recent speciation 629 associated with the evolution of selfing in Capsella. Proceedings of the National Academy of 630 Sciences of the USA 106: 5241-5245.

631 Gos G, Slotte T, Wright SI. 2012. Signatures of balancing selection are maintained at 632 disease resistance loci following mating system evolution and a population bottleneck in the 633 genus Capsella. BMC Evolutionary Biology 12: 152.

634 Gouy M, Guindon S, Gascuel O. 2010. SeaView version 4: A multiplatform graphical user 635 interface for sequence alignment and phylogenetic tree building. Molecular Biology and 636 Evolution 27: 221-224. 2009. Recent speciation of Capsella rubella from Capsella grandiflora, associated with loss 639 of self-incompatibility and an extreme bottleneck. Proceedings of the National Academy of 640 Sciences of the USA 106: 5246-5251.

641 Haldane JBS. 1927. A mathematical theory of natural and artificial selection, part V:

642 selection and mutation. Mathematical Proceedings of the Cambridge Philosophical Society $643 \quad 23: 838-844$.

644 Haley CS, Knott SA. 1992. A simple regression method for mapping quantitative trait loci in 645 line crosses using flanking markers. Heredity. 69: 315-324.

646 Harris, R.S. 2007. Improved pairwise alignment of genomic DNA. PhD Thesis, 647 Pennsylvania State University, Pennsylvania, USA.

648 Holt C, Yandell M. 2011. MAKER2: an annotation pipeline and genome-database 
650 Huang H-R, Liu J-J, Xu Y, Lascoux M, Ge X-J, Wright SI. 2018. Homeologue-specific 651 expression divergence in the recently formed tetraploid Capsella bursa-pastoris 652 (Brassicaceae). The New Phytologist 42: e46-635. Hurka H, Friesen N, German DA, Franzke A, Neuffer B. 2012. 'Missing link' species

654 Capsella orientalis and Capsella thracica elucidate evolution of model plant genus Capsella 655 (Brassicaceae). Molecular Ecology 21: 1223-1238.

656 Kacser H, Burns JA. 1981. The molecular basis of dominance. Genetics 97: 639-666.

657 Katoh K, Misawa K, Kuma K-I, Miyata T. 2002. MAFFT: a novel method for rapid 658 multiple sequence alignment based on fast Fourier transform. Nucleic Acids Research 30: $659 \quad 3059-3066$.

661 Long-term balancing selection drives evolution of immunity genes in Capsella. eLife 8: 662 e43606.

664 scalable and accurate long-read assembly via adaptive k-mer weighting and repeat separation. 665 Genome Research 27: 722-736.

666 Kusaba M, Nishio T, Satta Y, Hinata K, Ockendon D. 1997. Striking sequence similarity

667 in inter- and intra-specific comparisons of class I SLG alleles from Brassica oleracea and 668 Brassica campestris: Implications for the evolution and recognition mechanism. Proceedings 669 of the National Academy of Sciences of the USA 94: 7673-7678. 
671 C. 2018. Paternally expressed imprinted genes associate with hybridization barriers in

672 Capsella. Nature Plants 4: 352-357.

673 Larsson A. 2014. AliView: a fast and lightweight alignment viewer and editor for large 674 datasets. Bioinformatics. 30: 3276-3278.

675 Li H. 2013. Aligning sequence reads, clone sequences and assembly contigs with BWA676 MEM. arXiv:1303.3997v2, https://arxiv.org/abs/1303.3997v2.

677 Liu P, Sherman-Broyles S, Nasrallah ME, Nasrallah JB. 2007. A cryptic modifier causing 678 transient self-incompatibility in Arabidopsis thaliana. Current Biology 17: 824-824.

679 Llaurens V, Billiard S, Leducq J-B, Castric V, Klein EK, Vekemans X. 2008. Does

680 frequency-dependent selection with complex dominance interactions accurately predict allelic 681 frequencies at the self-incompatibility locus in Arabidopsis halleri? Evolution 62: 2545-2557.

682 Llaurens V, Gonthier L, Billiard S. 2009. The sheltered genetic load linked to the S locus in 683 plants: new insights from theoretical and empirical approaches in sporophytic self684 incompatibility. Genetics 183: 1105-1118.

685 Ma R, Han Z, Hu Z, Lin G, Gong X, Zhang H, Nasrallah JB, Chai J. 2016. Structural 686 basis for specific self-incompatibility response in Brassica. Cell Research 26: 1320-1329.

687 Mable BK, Hagmann J, Kim S-T, Adam A, Kilbride E, Weigel D, Stift M. 2017. What 688 causes mating system shifts in plants? Arabidopsis lyrata as a case study. Heredity 118: 110.

689 Mable BK, Schierup MH, Charlesworth D. 2003. Estimating the number, frequency, and

690 dominance of $S$-alleles in a natural population of Arabidopsis lyrata (Brassicaceae) with

691 sporophytic control of self-incompatibility. Heredity 90: 422-431. 
K, Altshuler D, Gabriel S, Daly M, et al. 2010. The Genome Analysis Toolkit: a quantifying mammalian transcriptomes by RNA-Seq. Nature Methods 5: 621-628.

Nasrallah JB, Nasrallah ME. 2014. $S$-locus receptor kinase signalling. Biochemical Society Transactions 42: 313-319.

Nasrallah JB, Liu P, Sherman-Broyles S, Schmidt R, Nasrallah ME. 2007. Epigenetic mechanisms for breakdown of self-incompatibility in interspecific hybrids. Genetics 175 : 1965-1973.

Nishio T, Kusaba M. 2000. Sequence Diversity of $S L G$ and $S R K$ in Brassica oleracea L . Annals of Botany 85: 141-146.

\section{Ossowski S, Schneeberger K, Lucas-Lledó JI, Warthmann N, Clark RM, Shaw RG,}

Weigel D, Lynch M. 2010. The rate and molecular spectrum of spontaneous mutations in Arabidopsis thaliana. Science 327: 92-94.

Pannell J, Barrett S. 1998. Baker's law revisited: Reproductive assurance in a metapopulation. Evolution 52: 657-668.

710 Porcher E, Lande R. 2005. The evolution of self-fertilization and inbreeding depression

711 under pollen discounting and pollen limitation. Journal of Evolutionary Biology 18: 497-508.

712 Porcher E, Lande R. 2005. Loss of gametophytic self-incompatibility with evolution of 713 inbreeding depression. Evolution 59: 46-60. 
Schoen DJ, Busch JW. 2009. The evolution of dominance in sporophytic self-

Schopfer CR, Nasrallah ME, Nasrallah JB. 1999. The male determinant of selfincompatibility in Brassica. Science 286: 1697-1700.

Shimizu KK, Tsuchimatsu T. 2015. Evolution of selfing: recurrent patterns in molecular adaptation. Annual Review in Ecology, Evolution, and Systematics 46: 593-622.

Lenhard M. 2016. Standing genetic variation in a tissue-specific enhancer underlies selfingsyndrome evolution in Capsella. Proceedings of the National Academy of Sciences of the USA. 113: 13911-13916.

Slotte T, Hazzouri KM, Ågren JA, Koenig D, Maumus F, Guo Y-L, Steige K, Platts AE,

Escobar JS, Newman LK, et al. 2013. The Capsella rubella genome and the genomic consequences of rapid mating system evolution. Nature Genetics 45: 831-835.

Slotte T, Hazzouri KM, Stern D, Andolfatto P, Wright SI. 2012. Genetic architecture and adaptive significance of the selfing syndrome in Capsella. Evolution 66: 1360-1374.

Smith TF, Waterman MS. 1981. Identification of common molecular subsequences. Journal of Molecular Biology 147: 195-197.

Stanke M, Steinkamp R, Waack S, Morgenstern B. 2004. AUGUSTUS: a web server for gene finding in eukaryotes. Nucleic Acids Research 32: W309-12. 
Steige KA, Laenen B, Reimegård J, Scofield DG, Slotte T. 2017. Genomic analysis reveals major determinants of cis-regulatory variation in Capsella grandiflora. Proceedings of the National Academy of Sciences of the USA. 114: 1087-1092.

Stein JC, Dixit R, Nasrallah ME, Nasrallah JB. 1996. SRK, the stigma-specific S locus receptor kinase of Brassica, is targeted to the plasma membrane in transgenic tobacco. The Plant Cell 8: 429-445.

Stein JC, Howlett B, Boyes DC, Nasrallah ME, Nasrallah JB. 1991. Molecular cloning of a putative receptor protein kinase gene encoded at the self-incompatibility locus of Brassica oleracea. Proceedings of the National Academy of Sciences of the USA 88: 8816-8820.

Takasaki T, Hatakeyama K, Suzuki G, Watanabe M, Isogai A, Hinata K. 2000. The S receptor kinase determines self-incompatibility in Brassica stigma. Nature 403: 913-916.

Takayama S, Shimosato H, Shiba H, Funato M, Che FS, Watanabe M, Iwano M, Isogai

A. 2001. Direct ligand-receptor complex interaction controls Brassica self-incompatibility. Nature. 413: 534-538.

Takayama S, Isogai A. 2005. Self-incompatibility in plants. Annual Review of Plant Biology 56: 467-489.

\section{Tarutani Y, Shiba H, Iwano M, Kakizaki T, Suzuki G, Watanabe M, Isogai A,}

Takayama S. 2010. Trans-acting small RNA determines dominance relationships in Brassica self-incompatibility. Nature 466: 983-986.

Tsuchimatsu T, Shimizu KK. 2013. Effects of pollen availability and the mutation bias on the fixation of mutations disabling the male specificity of self-incompatibility. Journal Of Evolutionary Biology 26: 2221-2232. 
760 locus in 1,083 Arabidopsis thaliana genomes. Molecular Biology and Evolution 34: 1878$761 \quad 1889$.

Tsuchimatsu T, Kaiser P, Yew C-L, Bachelier JB, Shimizu KK. 2012. Recent loss of selfincompatibility by degradation of the male component in allotetraploid Arabidopsis kamchatica. PLoS Genetics 8: e1002838.

Tsuchimatsu T, Suwabe K, Shimizu-Inatsugi R, Isokawa S, Pavlidis P, Städler T, Suzuki Arabidopsis by a mutation in the male specificity gene. Nature 464: 1342-1346.

Uyenoyama MK, Zhang Y, Newbigin E. 2001. On the origin of self-incompatibility haplotypes: transition through self-compatible intermediates. Genetics 157: 1805-1817. Jordan T, Shakir K, Roazen D, Thibault J, et al. 2013. From FastQ data to high confidence variant calls: the Genome Analysis Toolkit best practices pipeline. Current Protocols in Bioinformatics 11: 11.10.1-11.10.33.

Vekemans X, Poux C, Goubet PM, Castric V. 2014. The evolution of selfing from

775 outcrossing ancestors in Brassicaceae: what have we learned from variation at the $S$-locus?

776 Journal of Evolutionary Biology 27: 1372-1385.

777 Wright S. 1939. The distribution of self-sterility alleles in populations. Genetics 24: 538$778 \quad 552$.

779 Wright SI, Kalisz S, Slotte T. 2013. Evolutionary consequences of self-fertilization in 
780 plants. Proceedings of the Royal Academy of Sciences Series B Biological Sciences 280:

78120130133.

782 Ågren JA, Wang W, Koenig D, Neuffer B, Weigel D, Wright SI. 2014. Mating system

783 shifts and transposable element evolution in the plant genus Capsella. BMC genomics 15:

784602. 
Figure 1. Self-compatibility is dominant and maps to the $S$-locus.

a. Logarithm of odds (LOD) profile resulting from interval mapping of self-compatibility in an interspecific Capsella orientalis $\times$ Capsella grandiflora $\mathrm{F} 2$ population. The dotted and dashed lines indicates the $1 \%$ vs. $5 \%$ genome-wide permutation-based significance threshold. The red vertical line shows the location of the canonical Brassicaceae $S$-locus. The 1.5-LOD confidence interval ranges from position $6,241,223$ to $8,742,368$, whereas the $S$-locus is located between positions 7,523,602 and 7,562,919 on chromosome 7. b. Estimated quantitative trait locus (QTL) additive effect (red line) and dominance deviation (blue line) across chromosome 7. Light shaded regions indicate standard errors.

Figure 2. Sequence comparison of full-length $S$-haplotype sequences results in identification of a frameshift deletion in Capsella orientalis SCR.

a. Phylogram of $S R K$ sequences, showing the diversity of $S$-alleles among Brassicaceae and

800 the close similarity of $S R K$ in the Arabidopsis halleri S12-haplotype to the clade containing 801 Capsella grandiflora CgS12, Capsella orientalis and Capsella bursa-pastoris (B subgenome) sequences (marked by a brace). b. Maximum likelihood gene trees for three $S$-locus genes: $S R K, S C R$ and $U-B O X$ showing

804 the relationship between A. halleri S12, C. grandiflora CgS12, two C. orientalis and C. bursa805 pastoris (B subgenome) accessions.

806 c. Plot showing the percentage of sequence similarity (sequence conservation) between $C$. 807 grandiflora CgS12 and C.orientalis 1979/09 S-haplotypes. Gene positions are indicated by 808 grey bars. 
d. Alignment of SCR sequences from A. halleri S12, C. grandiflora CgS12, C. orientalis and

C. bursa-pastoris (B subgenome) S-haplotypes. A frameshift deletion in the coding sequence

811 (marked by a red arrow) is found in C. orientalis but not in the two SI species A. halleri and

812 C. grandiflora.

813 e. Predicted SCR amino acid sequences for A. halleri S12, C. grandiflora CgS12, C. orientalis

814 and $C$. bursa-pastoris (B subgenome). The predicted protein sequence of $C$. orientalis lacks

815 five conserved cysteine residues (indicated by black arrows and orange boxes). The position

816 of the frameshift deletion is marked by a red arrow.

818 Figure 3. Success of controlled crosses based on pollen tube germination assays. Arrows

819 point to pollen tubes growing through the style. Scale bars, $200 \mu \mathrm{m}$.

820 a. Self-pollination of Capsella grandiflora carrying $\mathrm{CgS} 12$ allele results in no pollen tube

821 growth (incompatible reaction), demonstrating functional self-incompatibility.

822 b. Pollination of $C$. grandiflora carrying $C g S 12$ with pollen from an individual carrying

823 different $S$-haplotypes results in pollen tube growth (compatible reaction).

c. Pollination of $C$. grandiflora carrying $C g S 12$ with pollen from Capsella orientalis results in

825 pollen tube growth (compatible reaction), demonstrating that $C$. orientalis SCR is not

826 functional.

827 d. Pollination of $C$. orientalis with pollen from a C. grandiflora carrying $C g S 12$ results in 828 pollen tube growth (compatible reaction).

Figure 4. A conserved, $S$-linked Capsella orientalis SRNA is associated with repression of

\section{Capsella grandiflora $S C R$ in $S$-locus heterozygotes.}

832 a. Read depth of $C$. orientalis expresses $S$-linked small RNAs (sRNAs) homologous to

833 Arabidopsis halleri S12 Ah12mirS3 in flower buds. The grey box indicates the length of the 
834 sRNA precursor region and the location of Ah12mirS3 24 bp sRNA with highest expression 835 in A. halleri S12 is indicated in red.

836 b. Expression (reads per kilobase of transcript per million mapped reads, RPKM) of 18-27 nt

837 sRNAs in the Ah12mirS3-like RNA precursor region in flower buds differs between Capsella

838 orientalis $\times$ Capsella grandiflora F2s with different $S$-locus genotypes (Kruskal-Wallis

$\left.839 \chi^{2}=7.830, P=0.012\right): " \mathrm{Cg} / \mathrm{Cg}$ " and " $\mathrm{Co} / \mathrm{Co}$ " are homozygous for the $C$. grandiflora or $C$.

840 orientalis $S$-allele respectively, wheras "Co/Cg" are heterozygous. Only homozygotes or

841 heterozygotes for the $C$. orientalis $S$-allele express sRNAs in the Ah12mirS3-like RNA

842 precursor region (Dunn's test $P<0.01$ for both comparisons $\mathrm{Cg} / \mathrm{Cg}$ vs. $\mathrm{Co} / \mathrm{Cg}$ and $\mathrm{Cg} / \mathrm{Cg}$ vs.

$843 \mathrm{Co} / \mathrm{Co})$.

844 c. mirS3 24-nt small RNA sequences of A. halleri S12 (Ah12mirS3) and C. orientalis

845 (ComirS3) and the predicted target in C. grandiflora Cg88.15 SCR, located 665 bp from exon

$846 \quad 1$ and 183 bp from exon 2.

847 d. Relative expression (RPKM) of C. grandiflora SCR (blue) and C. orientalis $S C R$

848 (turquoise) in flower buds of F2 individuals with different $S$-locus genotypes: " $\mathrm{Cg} / \mathrm{Cg}$ " and

849 "Co/Co" are homozygous for the C. grandiflora or C. orientalis $S$-allele respectively, wheras

850 "Co/Cg" are heterozygous. C. grandiflora $S C R$ is repressed in C. grandiflora/C. orientalis

851 heterozygotes (Kruskal-Wallis $\chi^{2}(2)=9.9383, P<0.01$, Dunn's test $\mathrm{Z}(2)=2.25, P=0.012$

852 for $\mathrm{Co} / \mathrm{Cg}$ vs $\mathrm{Cg} / \mathrm{Cg}$ ). Values for $C$. grandiflora are relative to the median $\mathrm{RPKM}$ of $C$.

853 grandiflora homozygotes, whereas those for $C$. orientalis $S C R$ are relative to the median

854 RPKM of C. orientalis homozygotes.

855

856 Figure 5. The timing of loss of self-incompatibility in Capsella orientalis

857 Phylogenetic tree showing relationships among $S$-haplotypes and estimates of the timing of 858 the loss of self-incompatibility (SI) in C. orientalis based on analyses in BEAST2. Green bars 
859 at nodes indicate $95 \%$ credible intervals of the time to the most recent common ancestor

860 (TMRCA). The TMRCA of C. grandiflora CgS12 and C. orientalis + C. bursa-pastoris B

861 represents an upper bound for the timing of loss of SI in C. orientalis. Because C. orientalis

862 was self-compatible when it contributed to the origin of C. bursa-pastoris, the TMRCA of $C$.

863 orientalis and C. bursa-pastoris B represents a lower bound on the timing of loss of SI. 
865 Additional supporting information may be found in the online version of this article.

866 Methods S1 Additional methodological detail on experiments and analyses.

867 Table S1 Information of plant accessions used in this study.

868 Table S2 Information on sequence length and coverage for SMRT and MiSeq sequencing of

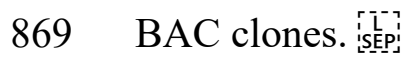

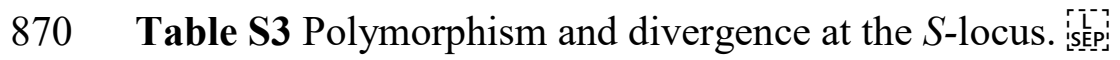

871 Table S4 Expression of SRK, SCR and U-box in flower buds and leaves of C. orientalis.

872 Table S5 Estimation of the timing of the loss of self-incompatibility performed in BEAST

873 with two competing models of population size change, constant and exponential.

874 Fig. S1 QTL mapping on different phenotyping scores.

875 Fig. S2 Sequence conservation (\% identity) between C. grandiflora CgS12 and A. halleri S12.

876 Fig. S3 Success of controlled crosses based on pollen tube germination assays. istepi]

877 Fig. S4 Number of pollen tubes in controlled crosses of C. orientalis, C. grandiflora

878 isE

879 Fig. S5 Qualitative RT-PCR demonstrating expression of SCR in flower buds of C. orientalis

880 and C. grandiflora individuals harboring $C g S 12$.

881 Fig. S6 Non-synonymous changes in the SRK hypervariable region. istep? 